\title{
The role of reading comprehension in mathematical modelling: improving the construction of a real-world model and interest in Germany and Taiwan
}

\author{
Janina Krawitz ${ }^{1}$ (D) $\cdot$ Yu-Ping Chang ${ }^{2} \cdot$ Kai-Lin Yang $^{3} \cdot$ Stanislaw Schukajlow $^{1}$
}

Accepted: 18 April 2021/ Published online: 20 May 2021

(C) The Author(s) 2021

\begin{abstract}
To solve mathematical modelling problems, students must translate real-world situations, which are typically presented in text form, into mathematical models. To complete the translation process, the problem-solver must first understand the real-world situation. Therefore, reading comprehension can be considered an essential part of solving modelling problems, and fostering reading comprehension might lead to better modelling competence. Further, ease of comprehension and involvement have been found to increase interest in the learning material, and thus, improving reading comprehension might also increase interest in modelling. The aims of this study were to (a) determine whether providing students with reading comprehension prompts would improve the modelling sub-competencies needed to construct a model of the real-world situation and their interest in modelling and (b) analyze the hypothesized effects in two different educational environments (Germany and Taiwan). We conducted an experimental study of 495 ninth graders (201 German and 294 Taiwanese students). The results unexpectedly revealed that providing reading comprehension prompts did not affect the construction of a real-world model. Further, providing reading comprehension prompts improved students' situational interest. The effects of providing reading comprehension prompts on the construction of a real-world model were similar in Germany and Taiwan. Students' interest in modelling improved more in Germany. An in-depth quantitative analysis of students' responses to reading prompts, their solutions, and their interest in the experimental group confirmed the positive relation between reading comprehension and modelling and indicated that the reading comprehension prompts were not sufficient for improving reading comprehension. Implications for future research are discussed.
\end{abstract}

Keywords Modelling competence · Reading comprehension · Interest C Comprehension questions · Country-specific differences $\cdot$ Word problems

Janina Krawitz

krawitz@uni-muenster.de

Extended author information available on the last page of the article 


\section{Introduction}

Mathematical modelling competence is an important part of mathematical literacy. However, research on modelling has demonstrated that students encounter various difficulties when solving modelling problems (Blum, 2015). Even at the beginning of the solution process, learners often struggle to understand the real-world situation and to structure and simplify the given information (Blum, 2015; Kintsch \& Greeno, 1985; Krawitz et al., 2017; Wijaya et al., 2014). In order to overcome these barriers, they need modelling sub-competencies to construct a structured and simplified mental representation of the real-world situation, here called the real-world model (Kaiser \& Brand, 2015). Consequently, teaching methods for modelling problems have often included elements that are aimed at improving the construction of a real-world model (Greefrath et al., 2018; Kaiser \& Brand, 2015; Schukajlow et al., 2012). Reading comprehension plays a decisive role in the construction of a real-world model (Leiss et al., 2010) because the modelling problems encountered in the classroom are often presented in text form. Also, the process of solving modelling problems in everyday life often includes gathering and interpreting information presented in text form (e.g., newspapers, timetables, books). Hence, reading comprehension is often required to understand the real-world situation, and consequently, interventions that address students' reading comprehension seem to be promising for fostering the ability to construct a realworld model and thereby improving one's overall modelling competence. However, there has not been much research that has focused on the effects of reading interventions on modelling competence and modelling sub-competencies. In particular, there has been a lack of experimental interventional studies in the field (Schukajlow et al., 2018).

Further, students' motivation plays a decisive role in the learning process in mathematics (Middleton \& Spanias, 1999; Schukajlow et al., 2017). One important motivational variable is students' interest in the learning material. Interest has been found to enhance students' learning, to predict academic decisions such as students' course choices in high school, and to have a positive effect on mathematics achievement (Heinze et al., 2005; Hidi \& Harackiewicz, 2000). Hence, it is important to investigate students' interest in modelling and to consider interventions for improving interest. One promising approach for triggering situational interest that we examined in our study involves facilitating the reading comprehension of the given texts or problems (Schraw et al., 1995; Wade et al., 1999).

The present article analyzes the effects of a reading intervention on students' modelling sub-competencies to construct a real-world model and on interest in two different educational environments. Prior research identified various perspectives on modelling, which differ in the aims they pursue with modelling and which can be related to different cultural backgrounds (Kaiser \& Sriraman, 2006). Also, the value assigned to modelling has been found to differ from country to country. We selected Germany and Taiwan because the educational environments in Germany and Taiwan are very different from each other. We targeted different educational environments in this study in order to determine whether the findings held for the different educational contexts that the students had been exposed to.

Following these considerations, the aims of the present study were (a) to test the effects of a reading intervention on the construction of a real-world model and on interest in modelling and (b) to examine whether the effects of the reading intervention on the construction of a realworld model and interest in modelling were similar in the two educational environments.

Further, we conducted an in-depth analysis of students who participated in the reading intervention. Thereby, we focused on students' reading comprehension ability and examined its relations to the construction of a real-world model and students' interest in modelling. 


\section{Modelling competence, interest in modelling, and reading comprehension in Germany and Taiwan}

\subsection{Modelling competence}

The core of mathematical modelling is the translation of a real-world problem into a mathematical model with the aim of solving the problem. The process of modelling is typically depicted as a cyclic process that moves from the real world to the mathematical world and back to the real world, passing through different phases that are required to solve the problem (see, e.g., Blum \& Leiss, 2007; Galbraith \& Stillman, 2006; Verschaffel et al., 2000). Demonstrating the willingness and ability to solve real-world problems through mathematical modelling is referred to as mathematical modelling competence (Kaiser, 2007). More specifically, we refer to an analytic understanding of modelling competence that is based on different sub-competencies (a description of different modelling strands can be found in Kaiser \& Brand, 2015). Modelling subcompetencies include - among metacognitive and social competencies-competencies that are related to the different phases of the modelling cycle (Kaiser, 2007; Maaß, 2006; Niss et al., 2007), namely: the competencies to (1) understand the real-world situation and construct an initial mental representation of the real-world situation (called the situation model); (2) structure and simplify the situation model, the resulting mental representation of which is referred to as the real-world model; (3) mathematize the realworld model, resulting in a mathematical model; (4) apply mathematical procedures to find a mathematical result; (5) interpret the mathematical result at the end of the solution process; and (6) validate the result with regard to the real-world situation.

\subsection{The modelling sub-competencies needed to construct a real-world model}

In the present article, we focus on the modelling sub-competencies needed to construct a realworld model. These sub-competencies are further explained and illustrated using the example of the Parachuting modelling problem presented in Fig. 1.

First, students have to construct a situation model. Therefore, they have to understand the information, presented here in the form of text that is accompanied by a table and a picture.

\footnotetext{
For parachuting, an airplane takes parachutists to an altitude of about 4,000 meters. From there, they jump off the plane.

Before a parachutist opens his/her parachute, he/she free falls about 3,000 meters. At an altitude of about 1,000 meters, the parachutist is carried off target by the wind. The horizontal shifts at different wind speeds are shown in the table below.
}

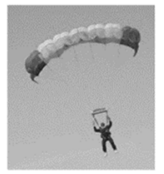

\begin{tabular}{|c|c|c|}
\hline $\begin{array}{c}\text { Wind } \\
\text { speed }\end{array}$ & $\begin{array}{c}\text { The horizontal shift per each thousand } \\
\text { meters of descent during free fall }\end{array}$ & $\begin{array}{c}\text { The horizontal shift per each thousand } \\
\text { meters of descent while gliding }\end{array}$ \\
\hline Light & 60 meters & 540 meters \\
\hline Medium & 160 meters & 1,440 meters \\
\hline Strong & 340 meters & 3,060 meters \\
\hline
\end{tabular}

What possible distance might the parachutist move during the entire jump, including free fall and gliding?

Fig. 1 The parachuting modelling problem adapted from Schukajlow and Krug (2014b, p. 500) 


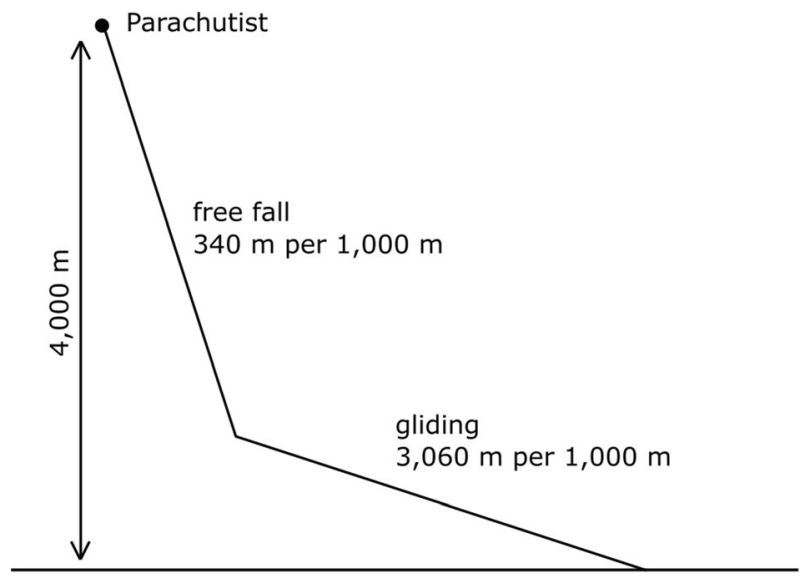

Fig. 2 Illustration of a real-world model for the parachuting problem under the assumption that a strong wind is blowing

Second, the learner has to transform his or her situation model into a real-world model (Fig. 2). This means the learner has to simplify the situation model by making an assumption about the wind speed (e.g., assume that there is a strong wind blowing). He or she has to structure the information by separating important from unimportant information (e.g., identifying the important information that the horizontal shift per each thousand meters of descent in strong wind conditions is $340 \mathrm{~m}$ during free fall and $3060 \mathrm{~m}$ while gliding) and construct relationships between the pieces of important information (e.g., connecting the information that the parachutist free falls about $3000 \mathrm{~m}$ to the information that the horizontal shift per each thousand meters is $340 \mathrm{~m}$ ).

Research on modelling has shown that solving modelling problems is demanding, and students are often already struggling at the beginning of the modelling process in trying to construct a real-world model (Blum, 2015; Kintsch \& Greeno, 1985; Krawitz et al., 2017; Leiss et al., 2010; Wijaya et al., 2014). In the study by Wijaya et al. (2014), more than one third of students' errors in solving modelling problems were related to the construction of a real-world model. These difficulties emphasize the need for research to examine interventions that can improve students' modelling sub-competencies to construct a real-world model while solving modelling problems.

\subsection{Students' interest in modelling}

Interest is considered a person-object relationship that refers to both the psychological state of attention and affect toward a particular topic (situational interest) and an enduring predisposition to reengage with the topic over time (individual interest) (Hidi \& Renninger, 2006). Interest is a domain- or content-specific motivational variable that combines affective and cognitive qualities (Harackiewicz et al., 2016; Schiefele et al., 1992). Theories of the development of interest propose that students pass through several phases as their interest develops: from unstable and triggered situational interest to stable and well-developed individual interest (Hidi \& Renninger, 2006). If a person repeatedly experiences situational interest with respect to a particular topic, he or she may also develop individual interest in the topic over time. Hence, the environment can contribute to the development of individual interest by stimulating situational interest and building on prior 
individual interest. For mathematical modelling, this means that if learners repeatedly experience situational interest when solving modelling problems, they are likely to develop individual interest in modelling. For modelling problems, different aspects can be sources of interest, namely, students may be interested in the process of modelling, the content, or the intramathematical problem. As affect and modelling competence are related to each other (Chamberlin, 2019; Schukajlow \& Krug, 2014a), enhancing interest in modelling is also beneficial for students' modelling competence. The important role that affect plays in modelling has been acknowledged in modelling research, and predictors such as authenticity, meaningfulness, and contexts have been discussed (Di Martino, 2019; Goldin, 2019). Several studies have addressed the question of how interest in solving mathematical problems can be enhanced. Building connections to reality is one approach that can be used to increase students' interest in mathematics because problem contexts can be a source of students' interest in working on the problems. However, the study conducted by Rellensmann and Schukajlow et al. (2017) showed that students do not perceive problems connected to reality per se as more interesting than intramathematical problems. In this line of research, studies have investigated whether personalizing the problems increases students' interest in working with the problems (Bates \& Wiest, 2004; Høgheim \& Reber, 2015). The results have shown that tailoring the context to students' personal interest has benefits for students' situational interest in working with the problems. However, personalized problems are often constructed with the help of digital tools and therefore are not easy to implement in classrooms. Our approach focuses on text-based interest (i.e., situational interest that comes from reading a text). As the problem is described in a textual format, we considered factors that are claimed to trigger text-based interest, such as meaningfulness, ease of comprehension, involvement, text cohesion, novelty, and emotiveness (Mitchell, 1993; Palmer, 2009; Schraw et al., 1995). Empirical results from factor analysis and correlational analysis have supported the importance of these sources of students' situational interest (Mitchell, 1993; Palmer, 2009; Schraw et al., 1995). For the present study, we consider involvement and ease of comprehension to be particularly important. Involvement refers to the extent to which students feel they are active participants. Ease of comprehension refers to how easy it is to understand a text. We discuss both sources in the context of reading comprehension and modelling in the next section.

\subsection{Reading comprehension and its impact on constructing a real-world model and on interest in modelling}

Reading comprehension is defined as the active process of building an adequate mental representation of a text (Durkin, 1993; Kintsch, 1986). Texts in mathematics often include discontinuous elements such as tables, figures, or formulae. If the text is accompanied by pictures, an integrated mental representation is built on the basis of the text and pictures (Schnotz \& Bannert, 2003). Reading comprehension can be claimed to be one of the sub-competencies needed to understand the real-world situation because the situation is often presented in a textual format in the classroom or in everyday contexts involving textual information, such as newspaper articles, product information, reports, and many others. Hence, reading comprehension can also be considered a sub-competency that is necessary for constructing a real-world model because structuring and simplifying the given information directly depend on an adequate understanding. The importance of reading comprehension for modelling has been acknowledged in research on modelling (Leiss et al., 2010; Leiss et al., 2019), and theoretical descriptions of the modelling process have been built on research on text comprehension (Kintsch \& Greeno, 1985). Empirical findings have supported the positive relation between reading comprehension and modelling 
competence (Krawitz et al., 2017; Leiss et al., 2010; Leiss et al., 2019; Vilenius-Tuohimaa et al., 2008). Hence, interventions that address students' reading comprehension seem to offer a promising approach for fostering modelling sub-competencies to construct a real-world model and thereby improving overall modelling competence. However, hardly any intervention studies have tried to enhance modelling competence by fostering reading comprehension, and the few existing ones have not been successful (Hagena et al., 2017; Krawitz et al., 2017). Thus, further investigations are necessary to identify the conditions under which reading interventions are beneficial for modelling. One approach for enhancing reading comprehension is to present questions that address important pieces of information and their relations given in the text, referred to here as reading comprehension prompts. The impact of questions on reading comprehension is widely acknowledged in reading research, and answering questions is considered an important strategy for boosting reading comprehension. In particular, reading research has shown that reading comprehension prompts can guide readers' attention to important aspects of the text (Ge \& Land, 2003) and increase their engagement with the text because the contents of the text are more actively processed when the reader has to answer questions about the contents (National Reading Panel, 2000). However, questions are not as beneficial per se. The impact strongly depends on factors such as the type of question (Cerdán et al., 2009) and readers' reading proficiency (van den Broek et al., 2001). Working on high-level questions was found to be more beneficial for reading comprehension than working on low-level questions (Cerdán et al., 2009). The results from the study by van den Broek et al. (2001) suggest that more proficient readers benefit more from questions, whereas less proficient readers can suffer from having to answer questions because of an increase in cognitive demand from having to think about them. However, these findings were based on scientific or narrative texts, and one question that remains unanswered is whether reading comprehension prompts are also beneficial for the text included in modelling problems. Research on modelling has provided initial indications that reading comprehension prompts might foster the construction of a real-world model and thereby enhance modelling competence. In the study conducted by Schukajlow et al. (2015) and similarly also in the study by Hankeln and Greefrath (2020), students received a scaffolding instrument called a solution plan to guide their modelling processes. The solution plan consisted of prompts referring to the different phases of the modelling cycle, including prompts to trigger reading comprehension ("Read the text precisely! Imagine the situation clearly!"). The results showed that using the solution plan was beneficial for students' modelling competence, but the specific role of reading comprehension prompts could not be derived from the data as it was not clear which prompts were responsible for the positive effect on modelling competence.

Further, reading comprehension prompts might increase students' interest in modelling because they address two important sources of situational interest: involvement and ease of comprehension. First, reading comprehension prompts might trigger involvement because, by working with the prompts, students become more actively involved in the reading process. Second, reading comprehension prompts might affect ease of comprehension because they are suggested to facilitate reading comprehension, and reading research has indicated that if texts become easier to understand, they are perceived as more interesting (Schraw et al., 1995; Wade et al., 1999). Modelling problems often place high demands on students' reading comprehension, and thus, their ease of comprehension may be compromised. This is a potential reason for the unexpected findings that students perceive modelling problems as similar to (Schukajlow et al., 2012) or even less interesting (Rellensmann \& Schukajlow, 2017) than problems with no connection to reality. Consequently, we expected that reading comprehension prompts would increase students' interest in modelling. 


\subsection{Educational environments in Germany and Taiwan}

One of the aims of the present study was to investigate the role of the educational environment the students had been exposed to. Comparing students from educational environments that are very different, such as Germany and Taiwan, can provide indications for the validity of the theoretically assumed relationships between reading comprehension, modelling competence, and interest in modelling. This section discusses differences in the educational environments of the two countries that we analyzed in the present study.

Students from East Asian countries - among them Taiwanese students-have been found to perform extremely well in international comparative studies of mathematics achievement such as TIMSS and PISA. However, there are some indications that modelling and applications play only minor roles in Taiwanese compared with German mathematics education. In Germany, modelling competence is embedded in the curriculum as one of six compulsory competencies (KMK, 2004), whereas it is not explicitly mentioned in the Taiwanese curriculum (Ministry of Education in Taiwan, 2003). Further, Taiwanese textbooks seem to focus on intramathematical tasks, as Taiwanese textbooks were found to contain the lowest proportion of real-world problems for geometry problems when compared with textbooks from Singapore, Finland, and the USA (Yang et al., 2017). This result is reflected in students' reports, as students from Taiwan reported that they encountered real-world problems in their math classes less often than German students (OECD, 2014). The few comparative studies that have analyzed students' achievement in modelling have pointed out that Western students are more experienced in solving modelling problems. Chang et al. (2020) showed that German students had higher modelling competence than Taiwanese students when the students from the two countries were on the same level of intramathematical competence. This difference was particularly remarkable for students with a low level of intramathematical competence.

Further, German and Taiwanese students' interest in modelling might also differ. Because they have less experience with modelling problems, Taiwanese students may find it more interesting to work on modelling problems than German students because novelty is an important source of situational interest (Palmer, 2009). However, more experience with modelling problems could also lead to greater interest in working on the problems because they might perceive the problems as more meaningful, which is also known as a source of situational interest (Mitchell, 1993). Little is known about differences in students' interest in modelling problems in different countries. As interest in modelling is related to interest in mathematics, the first indications of students' interest in modelling in Germany and Taiwan can be derived from the results of PISA 2012, where students' interest in mathematics was assessed. The results indicate that German students have a higher interest in mathematics compared with Taiwanese students (OECD, 2013), which is surprising given the much higher mathematical performance of Taiwanese learners. As cognitive and affective theories such as theories of modelling competence and theories of interest do not depend on education environments, we expected that reading comprehension prompts would have similar effects on the construction of a real-world model and interest in modelling in Germany and Taiwan.

\subsection{Hypotheses and path-analytical model}

On the basis of theoretical considerations and the prior empirical findings described above, we developed the following hypotheses: 
Hypothesis 1 (reading comprehension prompts)

The presentation of reading comprehension prompts will positively affect the modelling subcompetencies needed to construct a real-world model and students' interest in solving modelling problems:

a) Presenting reading comprehension prompts will lead to higher scores on the subcompetencies needed to construct a real-world model.

b) Presenting reading comprehension prompts will lead to a higher interest in solving modelling problems.

Hypothesis 2 (educational environment)

The effects of presenting reading comprehension prompts on the construction of a real-world model and on interest will be similar in both educational environments.

a) The effect of presenting reading comprehension prompts on the construction of a realworld model will be similar in the two educational environments (Germany and Taiwan).

b) The effect of presenting reading comprehension prompts on interest in modelling will be similar in the two educational environments (Germany and Taiwan).

The hypothesized path model (Fig. 3) links reading comprehension prompts - that were operationalized by presenting questions (reading comprehension prompts group vs. control group) - with the outcome measures (construction of a real-world model and interest in modelling) while controlling for intramathematical competence. Educational environment (Germany vs. Taiwan) was included as a moderator of the effects of reading comprehension prompts on the outcome variables.

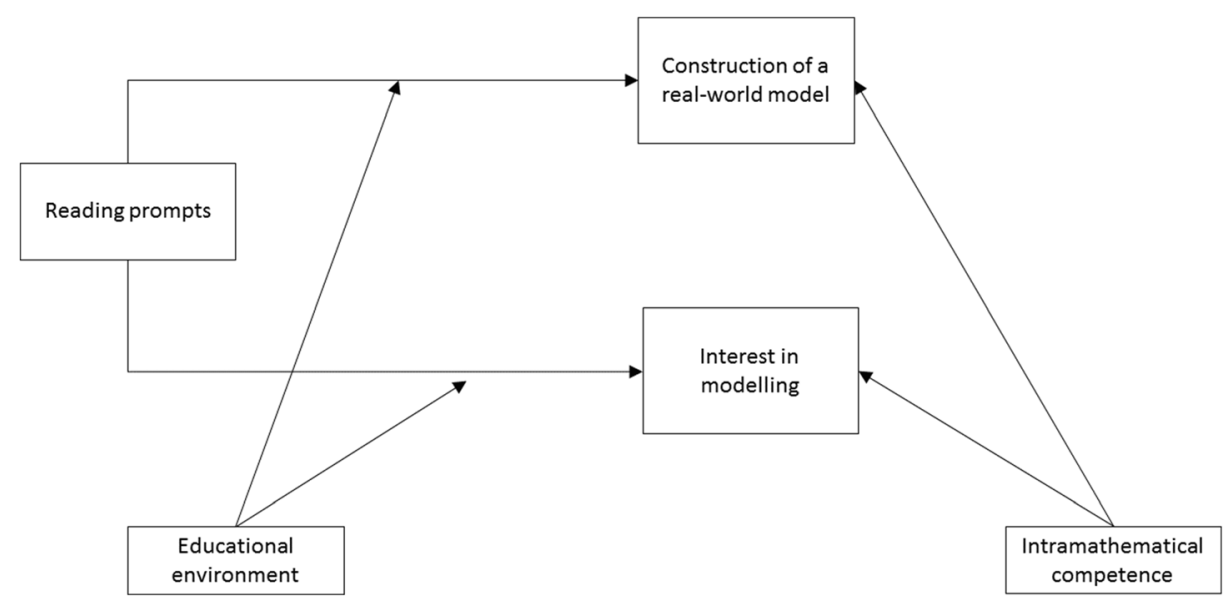

Fig. 3 Path-analytic model. Paths illustrate the direct effects of one construct (reading comprehension prompts) on the other construct (e.g., construction of a real-world model) or the moderating effects of a construct (educational environment) on the direct effects. The two paths from the control variable (intramathematical competence) to the outcomes illustrate that the investigated effects were controlled for intramathematical performance. 


\section{Method}

\subsection{Sample and procedure}

The present sample involved 495 ninth graders, including 201 German students from nine classes from high-track schools (German gymnasium; 50\% female, mean age $=14.96$ years) and 294 Taiwanese students from 12 classes in which all performance levels were taught (52\% female, mean age $=14.89$ years). Prior studies (e.g., Chang et al., 2020; OECD, 2019) have demonstrated that Taiwanese students have much higher intramathematical competence than German students. In order to balance these differences and improve the comparability of the groups regarding this important background variable, we collected data from German high-track schools and regular Taiwanese schools. We further compared the intramathematical competence of the two groups to control for whether the sampling strategy led to the intended result (see Section 4).

In each of the 21 classes, students were randomly assigned to an experimental condition (reading comprehension prompts group; RPG) or a control condition (control group; CG). Students in both conditions worked on a paper-and-pencil modelling test. In the RPG, reading comprehension prompts were used to trigger reading comprehension. Accordingly, students in the RPG received reading comprehension prompts that referred to the textual descriptions of the realworld situations (called situational descriptions). Students first read the situational description, then worked on two corresponding reading comprehension prompts, and subsequently worked on two modelling problems. Two sample pages from the RP test booklet from the context "Parachuting" are presented in the Appendix (Fig. 8). This procedure took $60 \mathrm{~min}$. After completing all tasks, students worked on the intramathematical problems for $20 \mathrm{~min}$. Students in the CG followed the same procedure, but they did not receive any reading comprehension prompts.

Reading comprehension prompts were operationalized as questions that referred to the information presented in the situational descriptions. The modelling problems were presented on a separate page after the reading comprehension prompts in order to reduce the risk that students would work on the modelling problems before answering the reading comprehension prompts. Responding to the reading comprehension prompts was aimed at helping students focus on important objects and on important relations between the elements given in the situational description. Consequently, one of the two questions for each situational description targeted important information, and the other question targeted relations between the given pieces of information. For example, for the parachuting situation, the situational description was the textual description of the parachutist's jump, including how he or she was carried off target by the wind (text and table presented in Fig. 1). The first reading comprehension prompt was "What is the horizontal shift per each thousand meters of descent while gliding when a parachutist is carried by a light wind?" (correct answer: "540 m"). This question referred to information provided in the table. The second reading comprehension prompt was "What is the horizontal shift per each thousand meters of descent when a parachutist is carried by a strong wind at about an altitude of 2,500 meters?" (correct answer: "340 m"). This question addressed the relations between and the interpretation of the given pieces of information. To respond, learners have to use the information given in the text to interpret the altitude of $2500 \mathrm{~m}$ as the free fall phase and then use the table to read out the horizontal shift for strong wind conditions during free fall.

The reading comprehension prompts were tested in a pilot study (Krawitz et al., 2017) and subsequently revised with a focus on the theoretically expected benefits of asking questions on reading comprehension. These benefits include addressing important pieces of information and their relations given in the text and thereby increasing students' engagement with the text (see Section 2.4). In the reading comprehension prompts, we decided also to address information that 
is not needed to solve the modelling problems because the aim of the prompts was to enhance the understanding of the situation and not to provide clues about which data should be used to solve the modelling problems.

\subsection{Measures}

\subsubsection{The modelling sub-competencies needed to construct a real-world model and intramathematical competence}

The modelling test included eight modelling problems that referred to the four situational descriptions (two modelling problems for each situational description). All situational descriptions used in the study were similar in length. Six modelling problems were adapted from previous studies (Blum, 2011; Schukajlow \& Krug, 2014b), and two modelling problems were developed in this study. The modelling problems could be solved with methods, such as applying the Pythagorean Theorem or by drawing a scaled diagram. The decision to limit the mathematical content area of the modelling problems was made to improve the fit between the modelling test and the intramathematical test. One modelling problem is presented in Fig. 1. Another modelling problem that referred to the same situational description was: "For his last jump, a parachutist glided about 1,600 meters after he had opened his parachute. Using the above clue, make reasonable assumptions about what kind of wind conditions most likely prevailed during this jump. Find a solution and clearly provide reasons for your answer."

In order to measure the modelling sub-competencies needed to construct a real-world model, students' solutions to the eight modelling problems were analyzed for whether the solution was based on a correct real-world model of the situation (scored 1) or not (scored 0). The use of problems that require students to work on all phases of the modelling process prevented us from asking questions that may have confused the students because they are not used to describe their construction of the real-world model. For example, for the modelling problem that went with the parachuting situation presented in Fig. 1 ("What possible distance might the parachutist move during the entire jump, including free fall and gliding?"), students had to make an assumption about the wind conditions and link this assumption to the information presented in the text and table (see Fig. 2). Figure 4 presents a student's solution that was scored as correct real-world model because the student selected the important information needed to solve the problem, made an assumption about the wind condition, and correctly assigned the data to the respective objects. The accuracy of the real-model was estimated based on students' written solutions (see Fig. 4).

In the solution presented in Fig. 5, the student assumed that a light wind was blowing but interpreted the side deviation as the distance traveled. Hence, the data were incorrectly assigned to the objects. Such a solution was scored as an incorrect real-world model.

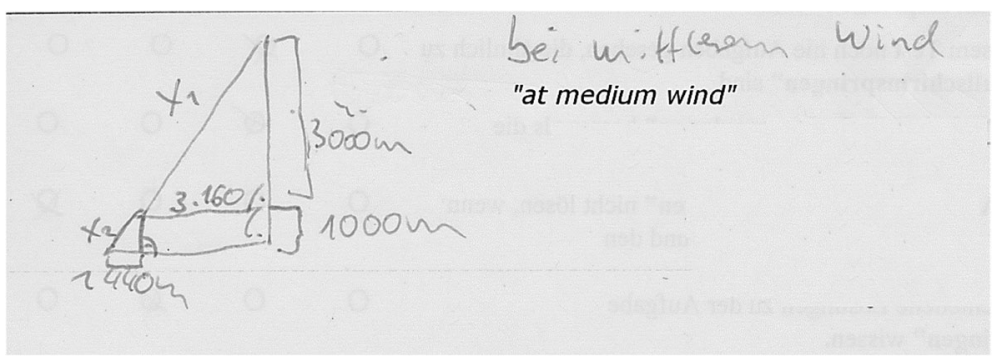

Fig. 4 Example of a student's solution that was scored as a correct real-world model 


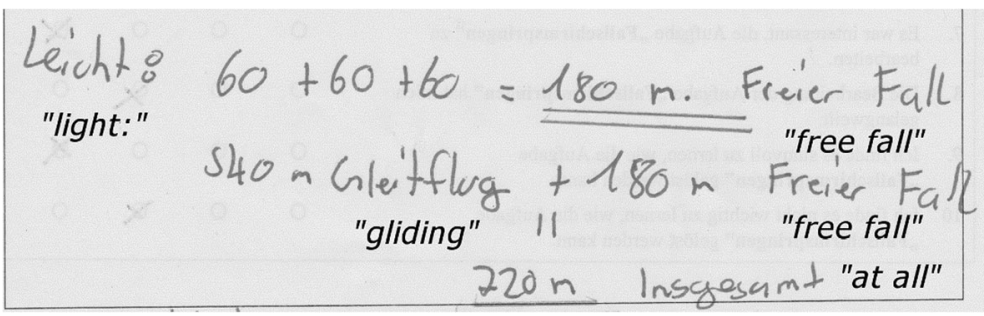

Fig. 5 Example of a solution that was scored as an incorrect real-world model

The scale reliability (Cronbach's alpha) for measuring the construction of a real-world model was 0.616. Two coders were involved in scoring the German test booklets, and six coders coded the Taiwanese part of the sample. At least $20 \%$ of the test booklets in each country were used to calculate intercoder reliability. Two coders scored the solutions for each item. The intercoder agreement between two coders (Cohen's K) was 0.694 or higher, indicating a substantial level of agreement. The coding was carried out by university students who completed a training and received a coding manual. When differences occurred, the coders discussed their judgments and made a consensual decision to choose one code.

Students' intramathematical competence was assessed as students' ability to solve intramathematical problems on the topic of the Pythagorean Theorem. On the intramathematical test, students were asked, for example, to calculate the length of the diagonal of a rectangle with a length of $3 \mathrm{~cm}$ and a width of $4 \mathrm{~cm}$ or to judge whether a given figure (nonright triangle) represents the Pythagorean Theorem. The scale consisted of 10 items, and its reliability (Cronbach's alpha) was 0.815 .

\subsubsection{Interest in solving modelling problems}

We used task-specific questionnaires in the present study in order to take into account the statelike nature of situational interest and the task-sensitivity of the construct (Knogler et al., 2015). We adapted the task-specific scale used in prior studies (Rellensmann \& Schukajlow, 2017; Schukajlow et al., 2012). For each of the four situational descriptions, after working on two modelling problems, students were asked whether they were interested in working on these problems. Using a 5-point Likert scale $(1=$ not at all true, $5=$ completely true $)$, students' responses indicated the extent to which they agreed with the following statement: "It was interesting to work on the problems '[Name of the situational description, e.g., Parachuting]'." The scale consisted of 4 items, and its reliability (Cronbach's alpha) was 0.835 .

\subsubsection{Translation of the material}

The material that was adopted from prior studies was translated into the English language. New material was directly developed in the English language. The English material was translated into German and Chinese. The second author of the paper, who understands all three languages, checked for the compatibility of the German and Chinese translations.

\subsection{Data analysis}

Means, standard deviations, and Pearson correlation coefficients, which are presented in Table 1, were calculated using SPSS. All estimation and data fitting procedures for testing the hypothesized 
Table 1 Means, standard deviations, and correlations of all variables

\begin{tabular}{|c|c|c|c|c|c|c|c|}
\hline Variable & $M$ & $S D$ & 1 & 2 & 3 & 4 & 5 \\
\hline 1 Construction of a real-world model & 0.123 & 0.165 & - & & & & \\
\hline 2 Intramathematical competence & 0.515 & 0.281 & $0.470^{* *}$ & - & & & \\
\hline 3 Interest in modelling & 2.781 & 0.950 & 0.079 & $0.191^{* *}$ & - & & \\
\hline 4 Reading comprehension prompts ${ }^{\mathrm{a}}$ & - & - & -0.025 & 0.012 & $0.090^{*}$ & - & \\
\hline 5 Educational environment ${ }^{\mathrm{a}}$ & - & - & $-0.256^{* *}$ & -0.013 & $0.180^{* *}$ & 0.001 & - \\
\hline
\end{tabular}

${ }^{a}$ Dummy coded (reading comprehension prompts: $\mathrm{RPG}=1, \mathrm{CG}=0$; educational environment: Taiwan $=1$, Germany $=0$ ).

${ }^{*} p<.05$, two-tailed. ${ }^{* *} p<.01$, two-tailed

path-analytic model (Fig. 3) were carried out with Mplus (Muthén \& Muthén, 1998-2017). The variance-covariance matrix was analyzed by using maximum-likelihood estimation with robust standard errors. The reported $p$ values for the effects of the reading intervention were one-tailed because our expectations were directional, but they were two-tailed for the effects of educational environment. To examine clustering effects produced by the nonindependence of students nested in classes $(n=21)$, we calculated the intraclass correlation coefficient (ICC) for intramathematical competence. The ICC ( 0.31$)$ indicated that the intramathematical competence of students from the same classes was more similar than that of students from different classes. Thus, we used the "TYPE = COMPLEX" Mplus analytic option to account for the clustering effects (Stapleton, 2006). The treatment variables were dummy coded $(\mathrm{RPG}=1$ and $\mathrm{CG}=0)$. The model included 13 free parameters and 495 participants. The ratio of participants to parameters was about $38(495 / 13)$ and hence above the critical value of 5 for obtaining solid results (Kline, 2005). The model was fully saturated so that the fit indices were noninformative (i.e., $\mathrm{CFI}=1$; $\mathrm{SRMR}=0$ ).

\section{Results}

\subsection{Overall results}

First, we conducted a preliminary analysis of the sample and tested it for differences between German and Taiwanese students and between the conditions (RPG and CG) in order to obtain some indication of the comparability of the groups. For intramathematical competence, the results indicated that there were no differences between the German and Taiwanese students (Germany: $M=0.520 S D=0.251$; Taiwan: $M=0.512 S D=0.300), t(473.266)=$ $0.305, p=0.760$, nor were there differences between the students from the different experimental conditions (RPG: $M=0.518 S D=0.284$; CG: $M=0.512 S D=0.279$ ), $t(493)=-0.269, p=0.788$. These results justified the randomized assignment of students to the reading comprehension prompts and control conditions in our sample. Further, this preliminary analysis indicated that German and Taiwanese students were comparable concerning an important cognitive prerequisite: students' intramathematical competence.

The estimates of the path model that we created to test our hypothesis were based on the correlation matrix presented in Table 1. Figure 6 presents a graphical representation of the estimates. The means, standard deviations, and correlations of the study variables are presented separately for each experimental condition (RPG and CG) and educational environment (Germany and Taiwan) in Table 3 in the Appendix. 


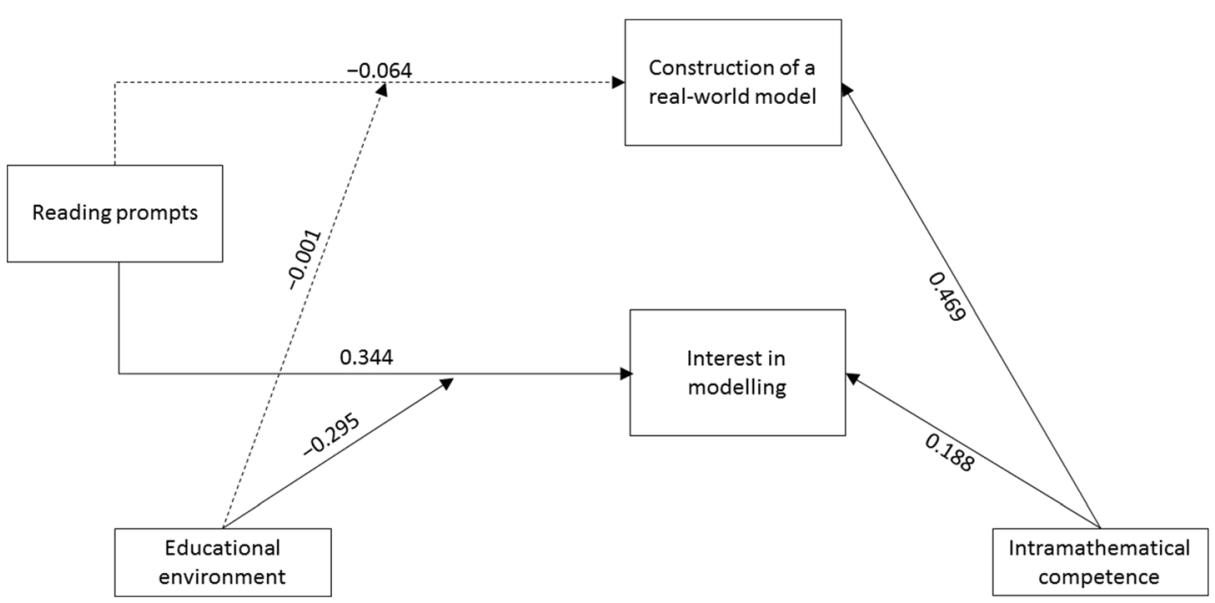

Fig. 6 Path model for testing the effects of presenting reading comprehension prompts on the construction of a real-world model and students' interest in modelling. Significant paths $(p<.05)$ are presented as solid lines and nonsignificant paths as broken lines (Estimates of the effects of binary covariates (reading prompts and educational environment) are standardized with respect to the dependent variables (STDY), and estimates of the effects of continuous covariates (intramathematical competence) are standardized with respect to both the covariate and the dependent variable (STDXY). STDY standardized regression coefficients $(\beta)$ can be interpreted as the predicted change in the (residualized) criterion measures (in standard deviation units) when the covariate (reading prompts or educational environment) changed by one unit. For example, if the value for reading prompts changes from 0 to 1 (change in the reading treatment condition), interest increases by $\beta \times \mathrm{SD}_{\text {Int }}$ $=0.344 \times \mathrm{SD}_{\text {Int }}$ STDXY is interpreted as the predicted change in standard deviation units when the covariate changes by one standard deviation)

\subsection{Effects of reading comprehension prompts on the construction of a real-world model and on interest in modelling}

Regarding our reading intervention, we expected positive effects of presenting reading comprehension prompts on the construction of a real-world model (Hypothesis 1a) and on students' interest in solving modelling problems (Hypothesis 1b). The analysis partially supported our hypothesis. Presenting reading comprehension prompts did not affect the construction of a real-world model $(\beta=-0.064, p=0.220$, one-tailed $)$, but it positively affected students' interest in solving modelling problems $(\beta=0.344, p<0.01$, one-tailed).

\subsection{Educational environment as a moderator of the effects of reading comprehension prompts}

We further expected that the hypothesized positive effects of presenting reading comprehension prompts on the construction of a real-world model (Hypothesis 2a) and on interest in modelling (Hypothesis $2 b$ ) would be similar for the two educational environments of the students. There were no country-specific differences in the effect of presenting reading comprehension prompts on the construction of a real-world model ( $\beta=-0.001, p=0.996$ ). Further, contrary to our expectations, the path analysis revealed an effect of the country on the effect of reading comprehension prompts on students' 
interest in modelling $(\beta=-0.295, p<0.05)$, indicating that presenting reading comprehension prompts is more beneficial for interest in solving modelling problems for German students than for Taiwanese students. An analysis of the effects of reading comprehension prompts on interest in the respective educational environment revealed significant positive effects in German but not in Taiwanese students (Germany: $\beta=$ $0.352, p<.01$; Taiwan: $\beta=0.051, p=0.309$ ).

\section{In-depth analysis of the reading comprehension prompts condition}

We conducted an in-depth analysis to investigate the role of reading comprehension in determining the modelling sub-competencies necessary to construct a real-world model and interest in modelling in the group of students who participated in the reading intervention. The aims were, first, to validate the positive relation between reading comprehension and modelling competence that is assumed in modelling theories and, second, to obtain an indication of why providing reading comprehension prompts only partly supported our hypotheses on the positive effects of reading comprehension prompts on the construction of a real-world model and interest in modelling. The research questions for the in-depth analysis were:

1. Is reading comprehension positively related to the construction of a real-world model and interest in modelling?

2. Are the effects of reading comprehension on the construction of a real-world model and interest in modelling similar in different educational environments?

\subsection{Method of the in-depth analysis}

To conduct our analysis, we assessed students' reading comprehension in addition to other measures. Reading comprehension was measured by scoring the answers to the reading comprehension prompts in the experimental condition $(N=245)$. Students received a score of 1 if they responded correctly to a reading comprehension prompt and a score of 0 if they responded incorrectly or did not responded at all (see the examples in Section 3.1). This scale ranged from 0 to 8 because we provided eight reading comprehension prompts in our study. The scale reliability (Cronbach's alpha) was 0.759 . Interrater agreement was calculated on a subset of at least $20 \%$ of the participants with sufficient agreement (Cohen's $k \geq 0.745$ ). The path model included 13 free parameters and 245 participants. Hence, the ratio of participants to parameters was above the critical value of 5 . We followed the same statistical approach that we used for the analysis of our primary hypotheses in the prior section.

\subsection{Results of the in-depth analysis}

The model parameter estimates were based on the correlation matrix presented in Table 2. Means, standard deviations, and correlations of all variables are also presented in this table. Figure 7 presents a graphical representation of the estimates. 
Table 2 Means, standard deviations, and correlations of all variables used in the in-depth analysis based on the students' responses in the RPG

\begin{tabular}{|c|c|c|c|c|c|c|c|}
\hline Variable & $M$ & $S D$ & 1 & 2 & 3 & 4 & 5 \\
\hline 1 Construction of a real-world model & 0.118 & 0.164 & - & & & & \\
\hline 2 Intramathematical competence & 0.518 & 0.284 & $0.446^{* *}$ & - & & & \\
\hline 3 Interest in modelling & 2.868 & 0.895 & 0.097 & $0.174^{* * *}$ & - & & \\
\hline 4 Reading comprehension & 0.712 & 0.247 & $0.339^{* * *}$ & $0.460^{* *}$ & $0.146^{*}$ & - & \\
\hline 5 Educational environment ${ }^{\mathrm{a}}$ & - & - & $-0.283^{* *}$ & -0.061 & 0.100 & $-0.269^{* *}$ & - \\
\hline
\end{tabular}

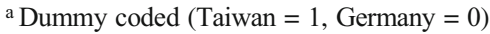

${ }^{*} p<.05$, two-tailed. ${ }^{* *} p<.01$, two-tailed

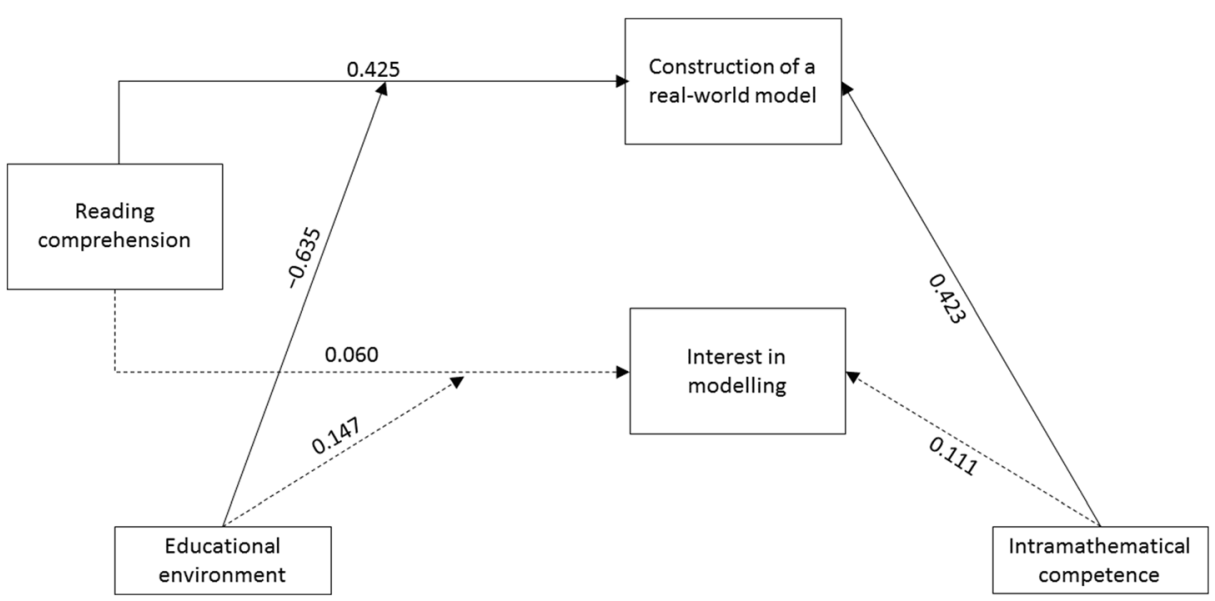

Fig. 7 Path model for testing the effects of reading comprehension on the construction of a real-world model and on students' interest in modelling. The analysis included only students from the experimental condition. Estimates are standardized (STDXY). Thus, the regression coefficients can be interpreted as the predicted change in the criterion measures in standard deviation units when the covariate changes by one standard deviation.

We found a positive impact of reading comprehension on the construction of a real-world model ( $\beta=0.425, p<0.01$, one-tailed). However, reading comprehension did not affect students' interest in solving modelling problems $(\beta=0.060, p=0.376$, one-tailed). Further, contrary to our expectations, educational environment was found to moderate the effect of reading comprehension on the construction of a real-world model $(\beta=-0.635, p<0.05)$. The analysis of the effects of reading comprehension on the construction of a real-world model and interest in modelling in the respective educational environment revealed that reading comprehension had a positive impact on the construction of a real-world model for German students ( $\beta=0.235, p<0.01$, one-tailed) but not for Taiwanese students $(\beta=-0.002, p=0.488$, one-tailed). The effect of reading comprehension on interest in modelling did not differ between German and Taiwanese students.

\section{Discussion}

In this study, we hypothesized and tested the effects of reading comprehension in two different educational environments on the modelling sub-competencies needed to construct a real-world model and students' interest in solving modelling problems while controlling for 
intramathematical competence. Reading comprehension was experimentally manipulated by providing students with reading comprehension prompts, operationalized as questions that addressed the real-world situations in the situational descriptions of the modelling problems. An in-depth analysis of the students who received reading comprehension prompts was conducted to investigate the impact of students' reading comprehension on the construction of a real-world model and interest in modelling in different educational environments and while controlling for students' intramathematical competence.

\subsection{Effects of reading comprehension on the construction of a real-world model and interest in modelling}

Contrary to our expectations, the construction of a real-world model was similar for students who were provided with reading comprehension prompts when compared with their peers who solved problems without reading comprehension prompts. The positive impact of questions on reading comprehension found in the domain of reading research (McKeown et al., 2009; Rickards, 1976) did not hold for students' mathematical modelling competence. An in-depth analysis of the relation between reading comprehension and modelling showed that students who correctly answered the reading comprehension questions were better at constructing a real-world model, indicating the importance of reading comprehension for modelling proposed in prior research. Apparently, providing reading comprehension prompts is not enough by itself. Rather, the quality of a student's engagement with the reading comprehension prompts, indicated by accurate answers, seems to enhance the modelling sub-competencies to construct a real-world model and thereby also enhances overall modelling competence. But why did the reading comprehension prompts fail to improve students' abilities to construct a real-world model in our study? A possible explanation is that students might have answered the reading comprehension prompts superficially without putting effort into reprocessing the text, and thereby, the benefits of presenting reading comprehension prompts could not take effect (Bråten et al., 2014; Pressley et al., 1989). Consequently, one implication from our study is that reading comprehension instructions should prompt students' ability to process the description of the real-world situation in the text (Pearson et al., 1992). We suggest that future studies should expand the presentation of reading comprehension prompts by teaching students how to use them in longer and more comprehensive interventions. Another reason could be that the reading comprehension prompts guided learners' attention to specific information and thereby did not enhance their understanding of the whole situation. It might be more beneficial to use more general reading comprehension prompts, such as "What is the text about? Write a short summary in your own words," or specifically for the parachuting situation, "Explain what horizontal shift means here and describe the factors that influence horizontal shift." Further, the cognitive cost of answering the prompts may have inhibited the positive effect of the reading comprehension prompts (van den Broek et al., 2001). These inhibiting effects might be particularly strong for readers with low proficiency levels who were not able to answer the questions. In addition, time constraints might have affected the results because students in the RPG had the same amount of test time as students in the CG.

The positive relation between reading comprehension and the construction of a real-world model found in our study adds to previous findings and indicates the importance of reading comprehension for modelling activities. The correlation of 0.339 found in our study 
is consistent with findings from previous studies (.198 in a study by Krawitz et al., 2017; .282 by Plath \& Leiss, 2018; and .486 by Leiss et al., 2010). Differences in the magnitudes of correlations between different studies can be explained by the specifics of the analysis. In the present study, we focused on the construction of a real-world model and not on modelling competence as a whole. Our study expanded the prior results by indicating the relevance of reading comprehension for the construction of a real-world model. However, future studies are necessary to investigate whether reading comprehension affects modelling or vice versa.

In line with our expectations, we found a positive effect of presenting reading comprehension prompts on students' interest in modelling. Even if presenting reading comprehension prompts did not directly improve modelling competence, it had a positive impact on students' perceptions of modelling. The in-depth analysis provided initial hints about the importance of different sources of interest in modelling. We found no effect of the accuracy of reading comprehension on students' interest in modelling. This finding indicates that ease of comprehension, which was found to be a source of situational interest in prior studies (Mitchell, 1993; Schraw et al., 1995), did not enhance interest in modelling in our studies. Consequently, other sources of situational interest such as students' level of involvement, which was triggered by the reading comprehension prompts, were potentially responsible for the positive effect on interest in modelling.

\subsection{Educational environment as a moderator of the effects of reading comprehension}

We collected data in Germany and Taiwan in order to validate the effects of a reading intervention on modelling and interest in modelling in two educational environments that are very different from each other. We expected that reading comprehension prompts would enhance modelling competence and interest in modelling for students in both educational environments.

There were no differences regarding the effect of presenting reading comprehension prompts on the construction of a real-world model. However, the in-depth analysis showed that reading comprehension is a significant predictor of the construction of a real-world model for German but not for Taiwanese students. One explanation for this result is that perhaps the Taiwanese students tended to fail to construct a real-world model even when they managed to understand the situation. In order to successfully construct a real-world model, students also need to structure and simplify the information, which also includes making assumptions. Students seem to lack meta-knowledge about modelling, particular the knowledge that solving modelling problems often requires learners to make assumptions (Krawitz et al., 2018), and it was found to be a particular strength of students educated in Germany compared with students educated in other countries. This is presumably because German students have more experience working with modelling problems (Chang et al., 2020; Hankeln, 2020).

The effect of presenting reading comprehension prompts on students' interest in modelling differed between the two educational environments such that the German students benefitted more from the reading comprehension prompts. A potential explanation is that different levels of interest in mathematics from German and Taiwanese students (OECD, 2013) caused this effect. Further studies should focus on the conditions in which fostering reading comprehension is beneficial for interest in modelling. 


\section{Limitations}

In the present study, reading comprehension was assessed by rating students' answers to the reading comprehension prompts. This allowed us to measure reading comprehension in a domain-specific way. For modelling, it is important that reading comprehension is measured in a mathematics-specific way (e.g., reading numerical information presented in tables) (Leiss et al., 2010), and hence, construct validity could be increased compared with the use of a general reading comprehension test. However, because of this assessment, we did not collect any information about reading comprehension in the control condition. Thus, we could not determine whether the reading comprehension prompts led to better reading comprehension in the experimental condition compared with the control condition.

Another limitation is that the scores for the construction of a real-world model were found to be low, which might have resulted in floor effects. However, solving modelling problems is known to be a demanding activity, and students' low scores on the construction of a real-world model reflect the use of demanding modelling problems to measure modelling competence. The construction of a real-world model was assessed by coding solutions to modelling problems. Results may have been different if the problems had focused on only this subcompetency. However, artificial tasks might have to be used if students are going to be asked to construct a real-world model. Further, the reading comprehension prompts and the modelling problems were presented on separate pages, but we do not know whether the students in the RP condition followed the given order and answered the reading comprehension prompts before working on the modelling problems. Future studies should include a treatment check.

Another limitation addresses the use of a questionnaire for measuring students' interest in modelling. Students were asked to rate how interesting it was to work on the respective task. We do not know which aspects of the tasks they referred to when they made their judgments. It is possible that different cultural and societal factors influenced the reports of the German and Taiwanese students, and thus, the comparison of these measures should be treated with caution. This measure also does not provide information about which aspects of the presented modelling problems the students referred to. German students might have referred to their interest in the real-world context, which is triggered by engagement with the reading comprehension prompts, whereas Taiwanese students might have referred to the task format of modelling problems themselves, which they found interesting because of its novelty. Further studies, particularly ones including qualitative approaches, are necessary to make more elaborative statements.

\section{Conclusion}

Our study shows that students' interest in modelling but not their modelling competence can be improved by presenting reading comprehension prompts. However, the findings differ for learners in Germany and Taiwan, indicating the relevance of educational environments for research in modelling. Consequently, reading comprehension is an essential but not sufficient condition for modelling. Students' experience with modelling seems to play a decisive role. Hence, we suggest that, in addition to students' reading comprehension, students' meta-knowledge about modelling, particularly the knowledge that modelling problems often require assumptions, should be addressed in modelling research and practice. 


\section{Appendix}

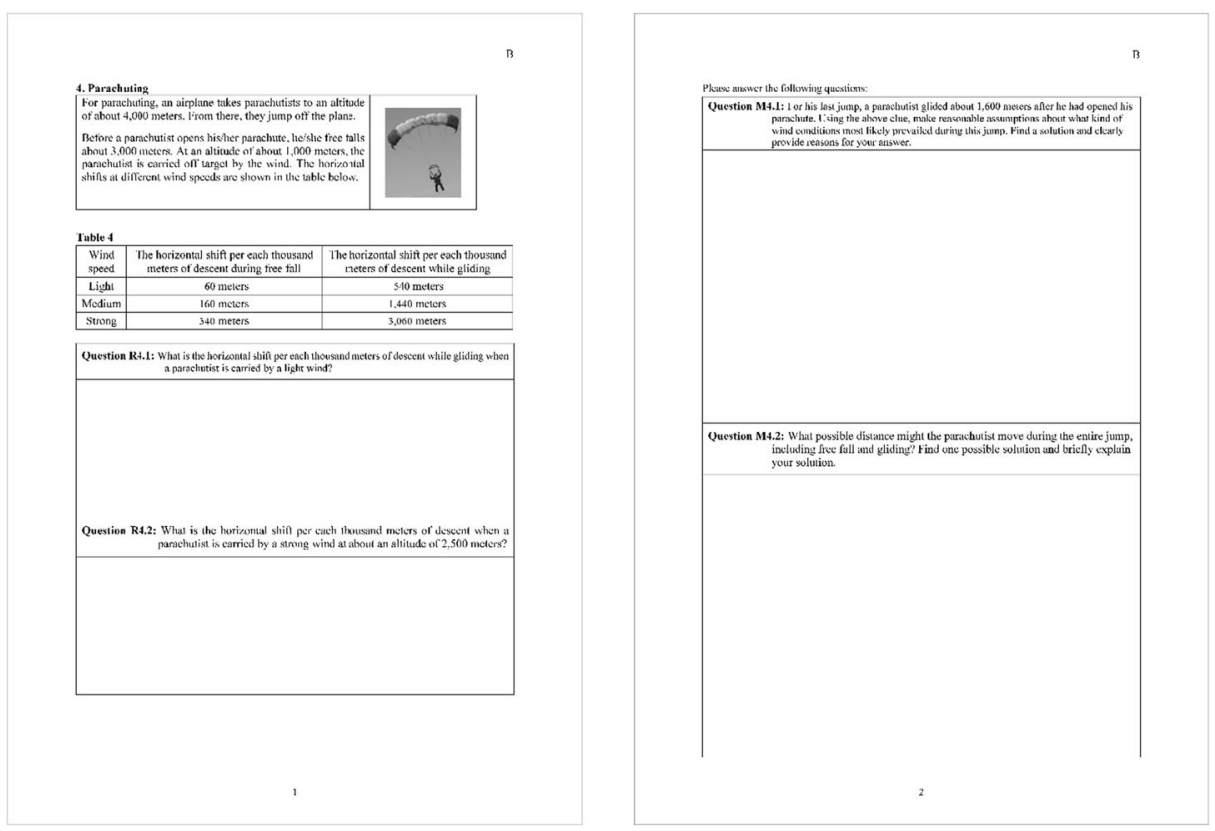

Fig 8 Sample pages from the test booklet for the RP condition, including the situational description, reading comprehension prompts, and modelling problems for the real-world situation parachuting.

Table 3 Means, standard deviations, and correlations presented separately for each experimental condition (RPG and CG) and educational environment (Germany and Taiwan)

\begin{tabular}{|c|c|c|c|c|c|c|c|}
\hline Group & Variable & $M$ & $S D$ & 1 & 2 & 3 & 4 \\
\hline \multirow[t]{4}{*}{ RP condition } & 1 Construction of a real-world model & 0.118 & 0.164 & - & & & \\
\hline & 2 Intramathematical competence & 0.518 & 0.284 & $0.446^{* *}$ & - & & \\
\hline & 3 Interest in modelling & 2.868 & 0.895 & 0.097 & $0.174^{* *}$ & - & \\
\hline & 4 Educational environment ${ }^{\mathrm{a}}$ & - & - & $-0.283^{* *}$ & -0.061 & 0.100 & - \\
\hline \multirow[t]{4}{*}{ Control condition } & 1 Construction of a real-world model & 0.127 & 0.166 & - & & & \\
\hline & 2 Intramathematical competence & 0.512 & 0.279 & $0.495^{* *}$ & - & & \\
\hline & 3 Interest in modelling & 2.697 & 0.995 & 0.068 & $0.205^{* *}$ & - & \\
\hline & 4 Educational environment ${ }^{\mathrm{a}}$ & - & - & $-0.231^{* *}$ & 0.034 & $0.251^{* *}$ & - \\
\hline \multirow[t]{4}{*}{ Germany } & 1 Construction of a real-world model & 0.174 & 0.175 & - & & & \\
\hline & 2 Intramathematical competence & 0.520 & 0.251 & $0.351^{* *}$ & - & & \\
\hline & 3 Interest in modelling & 2.576 & 0.949 & 0.086 & $0.144^{*}$ & - & \\
\hline & 4 Reading comprehension prompts ${ }^{\mathrm{a}}$ & - & - & 0.004 & 0.078 & $0.193^{* *}$ & - \\
\hline \multirow[t]{4}{*}{ Taiwan } & 1 Construction of a real-world model & 0.088 & 0.148 & - & & & \\
\hline & 2 Intramathematical competence & 0.512 & 0.300 & $0.584^{* *}$ & - & & \\
\hline & 3 Interest in modelling & 2.923 & 0.926 & $0.169^{* *}$ & $0.227^{* * *}$ & - & \\
\hline & 4 Reading comprehension prompts ${ }^{\mathrm{a}}$ & - & - & -0.050 & -0.026 & 0.021 & - \\
\hline
\end{tabular}

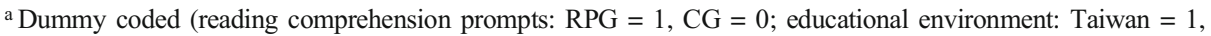
Germany $=0) . * p<.05$, two-tailed. $* * p<.01$, two-tailed 
Acknowledgments This study was conducted in the framework of the Taiwanese-German research program on cultural-societal influences on mathematics education (TaiGer).

Funding Open Access funding enabled and organized by Projekt DEAL. The meetings of the German and Taiwanese partners were funded by Deutsche Forschungsgemeinschaft (DFG) and the Ministry of Science and Technology Taiwan (MOST), allocated to Aiso Heinze (IPN Kiel, Germany) and Kai-Lin Yang (NTNU Taipei, Taiwan), respectively.

Open Access This article is licensed under a Creative Commons Attribution 4.0 International License, which permits use, sharing, adaptation, distribution and reproduction in any medium or format, as long as you give appropriate credit to the original author(s) and the source, provide a link to the Creative Commons licence, and indicate if changes were made. The images or other third party material in this article are included in the article's Creative Commons licence, unless indicated otherwise in a credit line to the material. If material is not included in the article's Creative Commons licence and your intended use is not permitted by statutory regulation or exceeds the permitted use, you will need to obtain permission directly from the copyright holder. To view a copy of this licence, visit http://creativecommons.org/licenses/by/4.0/.

\section{References}

Bates, E. T., \& Wiest, L. R. (2004). Impact of personalization of mathematical word problems on student performance. The Mathematics Educator, 14(2).

Blum, W. (2011). Can modelling be taught and learnt? Some answers from empirical research. In G. Kaiser, W. Blum, R. Borromeo Ferri, \& G. Stillman (Eds.), Trends in teaching and learning of mathematical modelling: ICTMA14 (pp. 15-30). Springer.

Blum, W. (2015). Quality teaching of mathematical modelling: What do we know, what can we do? In J. S. Cho (Ed.), Proceedings of the 12th International Congress on Mathematical Education (pp. 73-96). Springer.

Blum, W., \& Leiss, D. (2007). How do students and teachers deal with mathematical modeling problems? The example of Sugerloaf. In C. Haines, P. Galbraith, W. Blum, \& S. Khan (Eds.), Mathematical modelling: Education, engineering and economics - ICTMA12 (pp. 222-231). Horwood.

Bråten, I., Anmarkrud, Ø., Brandmo, C., \& Strømsø, H. I. (2014). Developing and testing a model of direct and indirect relationships between individual differences, processing, and multiple-text comprehension. Learning and Instruction, 30, 9-24.

Cerdán, R., Vidal-Abarca, E., Martínez, T., Gilabert, R., \& Gil, L. (2009). Impact of question-answering tasks on search processes and reading comprehension. Learning and Instruction, 19(1), 13-27.

Chamberlin, S. A. (2019). The construct of affect in mathematical modeling. In S. A. Chamberlin \& B. Sriraman (Eds.), Affect in Mathematical Modeling (pp. 15-28). Springer International Publishing.

Chang, Y.-P., Krawitz, J., Schukajlow, S., \& Yang, K.-L. (2020). Comparing German and Taiwanese secondary school students' knowledge in solving mathematical modelling tasks requiring their assumptions. ZDMMathematics Education, 52, 59-72.

Di Martino, P. (2019). The complex relationship between mathematical modeling and attitude towards mathematics. In S. A. Chamberlin \& B. Sriraman (Eds.), Affect in mathematical modeling (pp. 219-234). Springer International Publishing.

Durkin, D. (1993). Teaching them to read (Vol. 6). Allyn and Bacon.

Galbraith, P., \& Stillman, G. (2006). A framework for identifying student blockages during transitions in the modelling process. ZDM-Mathematics Education, 38(2), 143-162.

Ge, X., \& Land, S. M. (2003). Scaffolding students' problem-solving processes in an ill-structured task using question prompts and peer interactions. Educational Technology Research and Development, 51(1), 21-38.

Goldin, G. A. (2019). Exploring a conative perspective on mathematical engagement. In S. A. Chamberlin \& B. Sriraman (Eds.), Affect in Mathematical Modeling (pp. 111-129). Springer International Publishing.

Greefrath, G., Hertleif, C., \& Siller, H.-S. (2018). Mathematical modelling with digital tools — a quantitative study on mathematising with dynamic geometry software. ZDM-Mathematics Education, (50), 233-244.

Hagena, M., Leiss, D., \& Schwippert, K. (2017). Using reading strategy training to foster students' mathematical modelling competencies: Results of a quasi-experimental control trial. Eurasia Journal of Mathematics, Science and Technology Education, 13(7b), 4057-4085.

Hankeln, C. (2020). Mathematical modeling in Germany and France: A comparison of students' modeling processes. Educational Studies in Mathematics, 103(2), 209-229.

Hankeln, C., \& Greefrath, G. (2020). Mathematische Modellierungskompetenz fördern durch Lösungsplan oder Dynamische Geometrie-Software? Empirische Ergebnisse aus dem LIMo-Projekt [Fostering Mathematical 
Modelling Competency via Solution Plan or Dynamic Geometry Software? Empirical Results of the LIMoProject]. Journal für Mathematik-Didaktik. https://doi.org/10.1007/s13138-020-00178-9.

Harackiewicz, J. M., Smith, J. L., \& Priniski, S. J. (2016). Interest matters: The importance of promoting interest in education. Policy Insights From the Behavioral and Brain Sciences, 3(2), 220-227.

Heinze, A., Reiss, K., \& Rudolph, F. (2005). Mathematics achievement and interest in mathematics from a differential perspective. ZDM-Mathematics Education, 37(3), 212-220.

Hidi, S., \& Harackiewicz, J. M. (2000). Motivating the academically unmotivated: A critical issue for the $21 \mathrm{st}$ Century. Review of Educational Research, 70(2), 151-179.

Hidi, S., \& Renninger, K. A. (2006). The four phase model of interest development. Educational Psychologist, 41(2), 111-127.

Høgheim, S., \& Reber, R. (2015). Supporting interest of middle school students in mathematics through context personalization and example choice. Contemporary Educational Psychology, 42, 17-25.

Kaiser, G. (2007). Modelling and modelling competencies in school. In C. Haines, L. P. Galbraith, W. Blum, \& S. Kahn (Eds.), Mathematical Modelling: Education, Engineering and Economics - ICTMA12 (pp. 110119). Horwood.

Kaiser, G., \& Brand, S. (2015). Modelling competencies: Past development and further perspectives. In G. A. Stillman, W. Blum, \& M. S. Biembengut (Eds.), Mathematical modelling in education research and practice: Cultural, social and cognitive influences (pp. 129-149). Springer.

Kaiser, G., \& Sriraman, B. (2006). A global survey of international perspectives on modelling in mathematics education. ZDM-Mathematics Education, 38(3), 302-310.

Kintsch, W. (1986). Learning from Text. Cognition and Instruction, 3(2), 87-108.

Kintsch, W., \& Greeno, J. G. (1985). Understanding and solving word arithmetic problems. Psychological Review, 92(1), 109-129.

Kline, R. B. (2005). Principles and practice of structural equation modeling. Guilford Press.

KMK. (2004). Bildungsstandards im Fach Mathematik für den mittleren Schulabschluss [Intermediate-level education standards for mathematics]. Wolters Kluwer.

Knogler, M., Harackiewicz, J. M., Gegenfurtner, A., \& Lewalter, D. (2015). How situational is situational interest? Investigating the longitudinal structure of situational interest. Contemporary Educational Psychology, 43, 39-50.

Krawitz, J., Schukajlow, S., Chang, Y.-P., \& Yang, K.-L. (2017). Reading comprehension, enjoyment, and performance: How important is a deeper situation model? In B. Kaur, W. K. Ho, T. L. Toh, \& B. H. Choy (Eds.), Proceedings of the 41th Conference of the International Group for the Psychology of Mathematics Education (Vol. 3, pp. 97-104)

Krawitz, J., Schukajlow, S., \& Van Dooren, W. (2018). Unrealistic responses to realistic problems with missing information: What are important barriers? Educational Psychology, 38, 1221-1238.

Leiss, D., Schukajlow, S., Blum, W., Messner, R., \& Pekrun, R. (2010). The role of the situation model in mathematical modelling-task analyses, student competencies, and teacher interventions. Journal für Mathematik-Didaktik, 31(1), 119-141.

Leiss, D., Plath, J., \& Schwippert, K. (2019). Language and mathematics - key factors influencing the comprehension process in reality-based tasks. Mathematical Thinking and Learning, 21(2), 131-153.

Maßß, K. (2006). What are modelling competencies? ZDM-Mathematics Education, 38(2), 113-142.

McKeown, M. G., Beck, I. L., \& Blake, R. G. K. (2009). Rethinking reading comprehension instruction: A comparison of instruction for strategies and content approaches. Reading Research Quarterly, 44(3), 218-253.

Middleton, J. A., \& Spanias, P. A. (1999). Motivation for achievement in mathematics: Findings, generalizations, and criticisms of the research. Journal for Research in Mathematics Education, 30(1), 65-88.

Ministry of Education in Taiwan. (2003). Nine-year-integrated mathematics curriculum standards for national schools from grade 1 to 9 in Taiwan. MOE

Mitchell, M. (1993). Situational interest: Its multifaceted structure in the secondary school mathematics classroom. Journal of Educational Psychology, 85(3), 424-436.

Muthén, L. K., \& Muthén, B. O. (1998-2017). Mplus user's guide (8th ed.). Muthén \& Muthén.

Niss, M., Blum, W., \& Galbraith, P. L. (2007). Introduction. In W. Blum, P. L. Galbraith, H.-W. Henn, \& M. Niss (Eds.), Modelling and applications in mathematics education: The 14th ICMI Study (pp. 1-32). Springer.

OECD. (2013). PISA 2012 Results: Ready to learn: Students' engagement, drive and self-beliefs (Volume III). OECD Publishing.

OECD. (2014). PISA 2012 Results: What students know and can do - student performance in mathematics, reading and science (Volume I, Revised edition, February 2014). OECD Publishing.

OECD. (2019). PISA 2018 Results (Volume I). OECD Publishing.

Palmer, D. H. (2009). Student interest generated during an inquiry skills lesson. Journal of Research in Science Teaching, 46(2), 147-165. 
National Reading Panel. (2000). Teaching children to read: An evidence-based assessment of the scientific research literature on reading and its implications for reading instruction. National Institute of Child Health and Human Development.

Pearson, P. D., Roehler, L., Dole, J., \& Duffy, G. (1992). Developing expertise in reading comprehension. In S. J. Samuels \& F. A. E. (Eds.), What research says to the teacher (vol. 2, pp. 145-199). International Reading Association.

Plath, J., \& Leiss, D. (2018). The impact of linguistic complexity on the solution of mathematical modelling tasks. ZDM-Mathematics Education, 50(1-2), 159-171.

Pressley, M., Johnson, C. J., Symons, S., McGoldrick, J. A., \& Kurita, J. A. (1989). Strategies that improve children's memory and comprehension of text. The Elementary School Journal, 90(1), 3-32.

Rellensmann, J., \& Schukajlow, S. (2017). Does students' interest in a mathematical problem depend on the problem's connection to reality? An analysis of students' interest and pre-service teachers' judgments of students' interest in problems with and without a connection to reality. ZDM-Mathematics Education, 49(3), 367-378.

Rickards, J. P. (1976). Interaction of position and conceptual level of adjunct questions on immediate and delayed retention of text. Journal of Educational Psychology, 68(2), 210-217.

Schiefele, U., Krapp, A., \& Winteler, A. (1992). Interest as a predictor of academic achievement: A meta-analysis of research. In K. A. Renninger, S. Hidi, \& A. Krapp (Eds.), The role of interest in learning and developement (pp. 183-196). Erlbaum.

Schnotz, W., \& Bannert, M. (2003). Construction and interference in learning from multiple representation. Learning and Instruction, 13(2), 141-156.

Schraw, G., Bruning, R., \& Svoboda, C. (1995). Sources of Situational Interest. Journal of Reading Behavior, 27(1), 1-17.

Schukajlow, S., \& Krug, A. (2014a). Are interest and enjoyment important for students' performance? In C. Nicol, S. Oesterle, P. Liljedahl, \& D. Allan (Eds.), Proceedings of the Joint Meeting of PME 38 and PMENA 36 (vol. 5, pp. 129-136). PME.

Schukajlow, S., \& Krug, A. (2014b). Do multiple solutions matter? Prompting multiple solutions, interest, competence, and autonomy. Journal for Research in Mathematics Education, 45(4), 497-533.

Schukajlow, S., Leiss, D., Pekrun, R., Blum, W., Müller, M., \& Messner, R. (2012). Teaching methods for modelling problems and students' task-specific enjoyment, value, interest and self-efficacy expectations. Educational Studies in Mathematics, 79(2), 215-237.

Schukajlow, S., Kolter, J., \& Blum, W. (2015). Scaffolding mathematical modelling with a solution plan. ZDMMathematics Education, 47(7), 1241-1254.

Schukajlow, S., Rakoczy, K., \& Pekrun, R. (2017). Emotions and motivation in mathematics education: Theoretical considerations and empirical contributions. ZDM-Mathematics Education, 43(3), 307-322.

Schukajlow, S., Kaiser, G., \& Stillman, G. (2018). Empirical research on teaching and learning of mathematical modelling: A survey on the current state-of-the-art. ZDM-Mathematics Education, 50, 5-18.

Stapleton, L. M. (2006). An assessment of practical solutions for structural equation modeling with complex sample data. Structural Equation Modeling: A Multidisciplinary Journal, 13(1), 28-58.

van den Broek, P., Tzeng, Y., Risden, K., Trabasso, T., \& Basche, P. (2001). Inferential questioning: Effects on comprehension of narrative texts as a function of grade and timing. Journal of Educational Psychology, 93(3), 521-529.

Verschaffel, L., Greer, B., \& De Corte, E. (Eds.). (2000). Making sense of word problems. Swets \& Zeitlinger.

Vilenius-Tuohimaa, P. M., Aunola, K., \& Nurmi, E. (2008). The association between mathematical word problems and reading comprehension. Educational Psychology, 28(4), 409-426.

Wade, S. E., Buxton, W. M., \& Kelly, M. (1999). Using think-alouds to examine reader-text interest. Reading Research Quarterly, 34(2), 194-216.

Wijaya, A., van den Heuvel-Panhuizen, M., Doorman, M., \& Robitzsch, A. (2014). Difficulties in solving contextbased PISA mathematics tasks: An analysis of students' errors. The Mathematics Enthusiast, 11(3), 555-584.

Yang, D.-C., Tseng, Y.-K., \& Wang, T.-L. (2017). A comparison of geometry problems in middle-grade mathematics textbooks from Taiwan, Singapore, Finland, and the United States. EURASIA Journal of Mathematics Science and Technology Education, 13(7), 2841-2857.

Publisher's note Springer Nature remains neutral with regard to jurisdictional claims in published maps and institutional affiliations. 


\section{Affiliations}

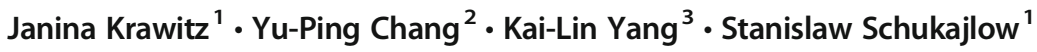

1 University of Münster, Münster, Germany

2 National Pingtung University, Pingtung, Taiwan

3 National Taiwan Normal University, Taipei, Taiwan 